\title{
Oscillations of Magnetospheric Boundaries Driven by IMF Rotations
}

\author{
H. Laakso, ${ }^{1}$ D. H. Fairfield ${ }^{2}$ M. R. Collier ${ }^{2}$ H. Opgenoorth,${ }^{1,3}$ T.-D. Phan, ${ }^{4}$ \\ D. G. Sibeck, ${ }^{5}$ B. L. Giles ${ }^{6}$ H. J. Singer, ${ }^{7}$ R. P. Lepping, ${ }^{2}$ R. P. Lin, ${ }^{4}$ F. S. \\ Mozer, ${ }^{4}$ R. F. Pfaff, ${ }^{2}$ K. Tsuruda, ${ }^{8}$ and J. R. Wygant ${ }^{9}$
}

\begin{abstract}
The arrival of the magnetic cloud on January 10, 1997, 01:05 UT, was preceded by a 12-hour interval of increased fluctuations in the IMF orientation, which drove boundary oscillations in the magnetosphere. The GEOTAIL spacecraft, initially located near the dawnside magnetopause, experienced multiple crossings of the magnetopause; at the geosynchronous distance, GOES 9 observed weak perturbations associated with these oscillations. During this time, the POLAR spacecraft encountered the dense high-latitude boundary layer, followed by several rapid encounters with the tenuous lobe. The boundary between the mantle and the lobe was observed to be thin as the crossings lasted only 30 seconds. The source of these boundary oscillations is magnetosheath pressure variations produced by rapid IMF directional changes.
\end{abstract}

\section{Introduction}

The outer regions of the magnetosphere, the magnetopause and the boundary layers, are continuously and directly under the influence of the shocked solar wind (called the magnetosheath) which drives a large variety of physical processes at these regions. The magnetopause is a transition layer between the magnetosheath and the magnetosphere where the magnetosheath pressure equals the magnetospheric pressure. Therefore magnetosheath pressure variations can move the magnetopause back and forth with respect to the Earth [Song et al., 1988; Sibeck et al., 1991].

Magnetosheath pressure variations can often be generated in the bow shock region (e.g., during variable IMF conditions) [Fairfield et al., 1990; Lin et al., 1996]. In particular, Fairfield et al. observed that upstream pressure disturbances convect through the bow shock and impinge on the magnetopause, and therefore rotational IMF discontinuities at the bow shock are transformed into magnetosheath pressure pulses (see also numerical simulations by Lin et al.). On the other hand it has been observed that IMF $B_{z}$ can affect the magnetopause position so that when IMF $B_{z}$ is negative, the magnetopause tends to move closer to the Earth [Sibeck et al., 1991; Petrinec and Russell, 1993].

\footnotetext{
${ }^{1}$ Finnish Meteorological Institute, Helsinki, Finland.

${ }^{2}$ NASA Goddard Space Flight Center, Greenbelt, MD.

${ }^{3}$ Also at Institut för Rymdfysik, Uppsala, Sweden.

${ }^{4}$ Space Sciences Lab., Univ. California, Berkeley, CA.

${ }^{5}$ The Johns Hopkins University, Laurel, MD

${ }^{6}$ NASA Space Flight Center, Huntsville, Alabama

${ }^{7}$ Space Environment Center, NOAA, Boulder, CO.

${ }^{8}$ ISAS, Sagamihara, Japan.

${ }^{9}$ Univ Minnesota, Minneapolis, MN.

Copyright 1998 by the American Geophysical Union.
}

Paper number 98GL50916.

0094-8534/98/98GL-50916\$05.00
During a quasiparallel bow shock, observations show the magnetosheath to be particularly turbulent, and a large variety of waves can exist near the magnetopause [Anderson, 1995]. Phan et al. [1994] observed that the magnetosheath plasma characteristics (e.g., density and flow) near the magnetopause can depend on magnetic shear, i.e., the angle between the local magnetosheath and the magnetopause magnetic fields.

Earthward of the magnetopause there exist boundary layers which are filled primarily with ions and electrons of solar wind origin. The boundary layer near the equator is called the low-latitude boundary layer (LLBL), where the plasma properties are known to be dependent on the magnetic shear across the magnetopause [Phan and Paschmann, 1996]. At higher latitudes, a boundary layer, called the plasma mantle, is located tailward of the cusp [Rosenbauer et al., 1975], although this region is not well understood, as it has been observed by only a small number of high-inclination, highaltitude satellites. During the ISTP era, the plasma mantle can be investigated in great detail with the POLAR satellite.

In this study we investigate a 12-hour interval on January 9, 1997, during which the IMF orientation varied strongly and caused prolonged oscillations of the outer boundaries in the magnetosphere. Not only was the magnetopause oscillating but the plasma mantle was in motion as well. These oscillations were observed by both GEOTAIL near the dawnside magnetopause and POLAR in the mantle/lobe regions. The magnetopause oscillations also caused weak signatures at the dayside geosynchronous distance observed by GOES 9 . During these boundary oscillations, the solar wind was monitored by the WIND satellite.

\section{Instrumentation}

This study presents data from four spacecraft (WIND, GEOTAIL, POLAR, and GOES 9) during the 12-hour interval before the arrival of the magnetic cloud at 01:05 UT on January 10, 1997. At that time, WIND was located in the upstream solar wind (appr. $X_{g s e}=80 R_{E}$ and $Y_{g s e}=-56 R_{E}$ ) while the other spacecraft were closer to the Earth. GOES 9 is a geosynchronous satellite located at about $135^{\circ}$ geographic west longitude (i.e., the local time is UT -9 hours). Figure 1a shows the GEOTAIL orbit in the $x y$ plane, and the positions of the magnetopause (solid line) and the bow shock (dashed line) for average solar wind conditions (dynamic pressure $=2.1 \mathrm{nPa}, \mathrm{IMF} B_{z}=0$ ). GEOTAIL is expected to be very close to the dawnside magnetopause. Figures $1 \mathrm{~b}$ and $1 \mathrm{c}$ illustrate the POLAR orbit in the $x z$ and $x y$ planes of the GSM coordinate system, respectively, indicating that the spacecraft was moving over the northern polar cap from dusk to dawn. 


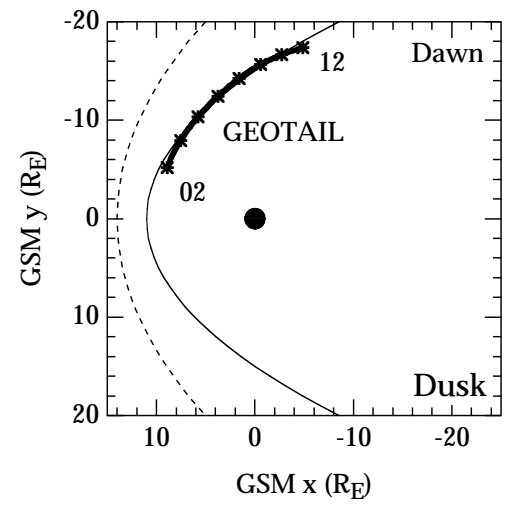

(a)

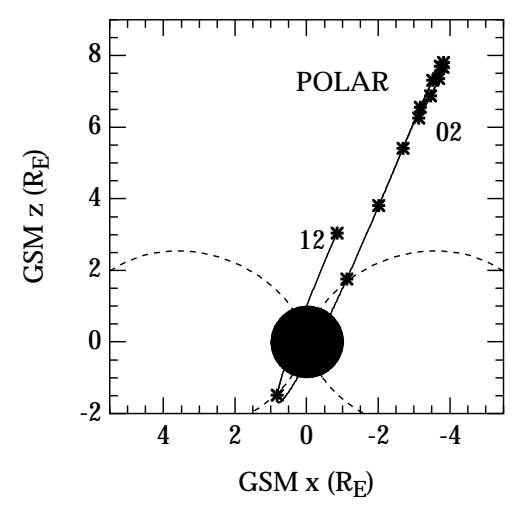

(b)

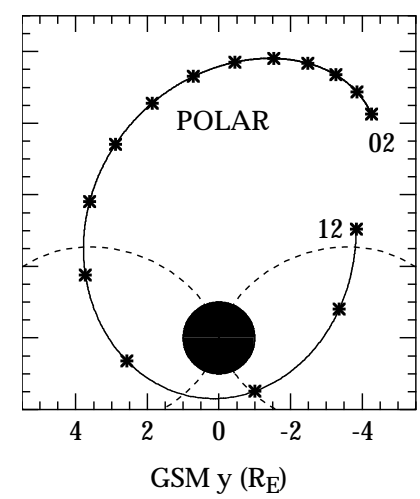

(c)

Figure 1. GEOTAIL AND POLAR positions from 12 UT on Jan 9 to 02 UT on Jan 10, 1997 in GSM coordinates. (a) GEOTAIL in the $x y$ plane; asterisks give GEOTAIL's position every two hours; POLAR in the (b) $x z$ and (c) $y z$ planes; asterisks show POLAR's position every one hour.

In this paper we utilize spacecraft potential measurements from the double probe experiments on the GEOTAIL and POLAR spacecraft. These experiments monitor the potential difference $\Delta V$ between an electric field probe $\left(V_{p}\right)$ and the spacecraft $\left(V_{s}\right)$, i.e., $\Delta V=V_{p}-V_{s}$, which is well correlated with the ambient electron density $N_{e}$ in tenuous plasmas [Laakso and Pedersen, 1997]. This is because $V_{p}$ is kept close to the plasma potential by means of a bias current. Hence, $\Delta V$ is approximately $-V_{s}$ which is, on the other hand, sensitive to $N_{e}$ as long as $N_{e}$ is less than a few hundred electrons per $\mathrm{cm}^{3}$ [Laakso and Pedersen, 1997].

\section{Observations}

Figure 2 presents data from WIND, GEOTAIL, and POLAR during the interval from 12:00 UT on January 9, 1997, to 02:00 UT on January 10, 1997. The top five panels taken from WIND show the solar wind dynamic pressure, measured by the $3 \mathrm{DP}$ experiment, the magnitude, polar angle $(\theta)$, and azimuthal angle $(\varphi)$ of the IMF, and the angle $\theta_{B n}$ between the IMF and the bow shock normal. The interplanetary shock associated with the magnetic cloud passes the spacecraft at 00:52 UT on January 10, 1997, and reaches the Earth 13 minutes later. The propagation time from WIND to GEOTAIL is expected to be 19 minutes which suggests that the cloud front was tilted with the Sun-Earth axis by $\sim 38^{\circ}$. The shock is preceded by a 12 -hour interval of increased fluctuations in the IMF orientation.

The dynamic pressure does not vary much, although some weak fluctuations are visible in the data, whereas the IMF rotates continuously after 13:47 UT. Large oscillations in $\theta_{B n}$ change the bow shock between quasiparallel $\left(\theta_{B n}<45^{\circ}\right)$ and quasiperpendicular $\left(\theta_{B n}>45^{\circ}\right)$ states which affect the level of turbulence and plasma pressure in the magnetosheath.

During this interval the GEOTAIL spacecraft was located near the dawnside magnetopause (see Figure 1a). The second panel from bottom of Figure 2 shows an increase in $\Delta V$ (i.e., thermal density) at 14:12 UT, when GEOTAIL suddenly moves into the magnetosheath. During the following two hours, the spacecraft has multiple magnetopause crossings. After 16:00 UT, GEOTAIL remains in the magnetosheath for several hours, which may be due to a slightly higher solar wind pressure, but more probably because $\theta_{B n}>45^{\circ}$, which may increase the magnetosheath pressure on the magnetopause.

After 19:50 UT the IMF direction shows a number of rapid directional changes, making the IMF more parallel to the bow shock normal. These events are followed by several additional magnetopause encounters by GEOTAIL. The GEOTAIL observations of the magnetopause oscillations are finally interrupted by the arrival of the interplanetary shock at 01:05 UT on Jan 10 which pushes the magnetopause closer to the Earth.

The bottom panel in Figure 2 shows $\Delta V$ (thermal density) measurements from the POLAR spacecraft. At 16:35 UT POLAR crosses the plasmapause (see Figure 1b and 1c), and a little later enters the northern lobe, where $\Delta V$ is about -30 volts, corresponding to $N_{e} \sim 0.1 \mathrm{~cm}^{-3}$ (Wygant et al., manuscript in preparation, 1997). At 17:58 UT, $\Delta V$ suddenly rises up to --12 volts $\left(N_{e} \sim 0.5 \mathrm{~cm}^{-3}\right)$ in 30 seconds. At the same time, the TIDE instrument on POLAR stops detecting low-energy ions (energy below $300 \mathrm{eV}$ ); partly due to high spacecraft potential values. These data suggest that the spacecraft moves into a relatively dense and hot plasma region, which is probably the plasma mantle rather than the cusp. After $20 \mathrm{UT} N_{e}$ begins to oscillate so that POLAR encounters the lobe several times. These rapid oscillations can be explained by means of earthward and anti-earthward motions of the plasma mantle due to magnetosheath pressure decreases and increases, respectively. After 22:20 UT $N_{e}$ remains lower and the spacecraft ceases enteries into the plasma mantle any more. At that time POLAR is already moving equatorward. Notice that the arrival of the shock at 01:05 UT cannot clearly be detected by the POLAR EFI experiment.

\section{Discussion}

During 12 hours prior to the arrival of the magnetic cloud at 01:05 UT on January 10, 1997, the IMF rotates strongly and causes oscillations of magnetospheric boundaries. Magnetopause motions are observed by GEOTAIL and oscillations of the plasma mantle are detected by POLAR. 


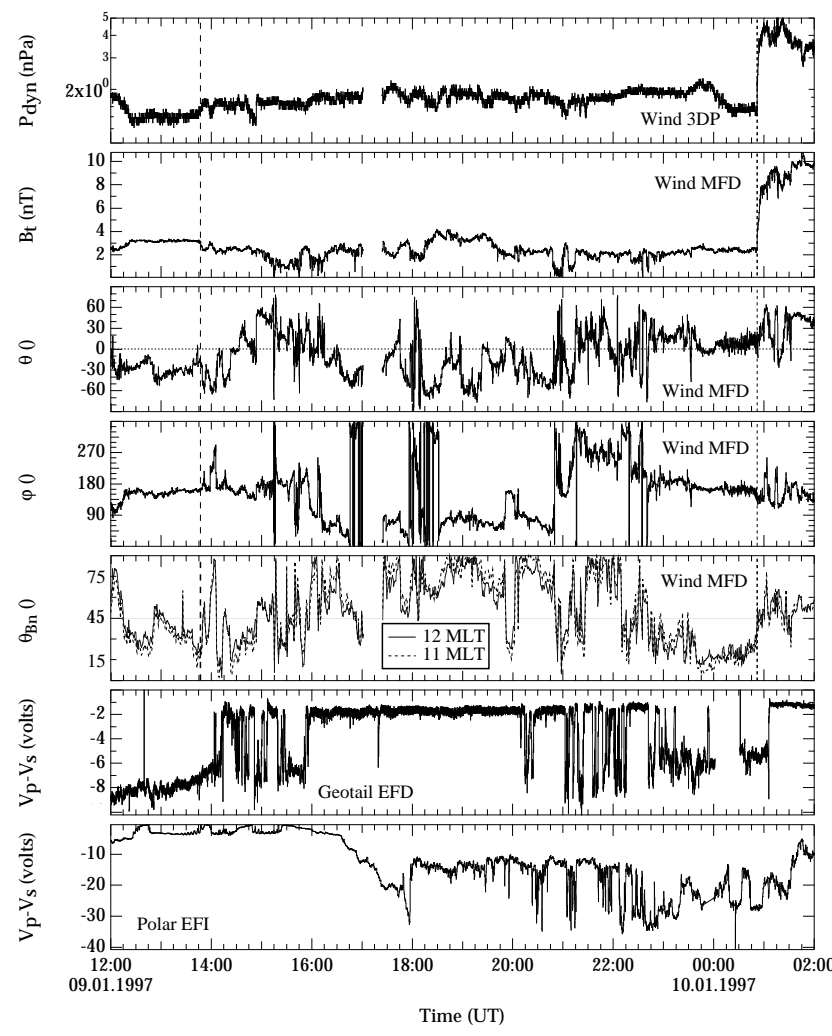

Figure 2. Solar wind fluctuations and boundary oscillations in the Earth's magnetosphere during the interval from January 9, 1997, 12:00 UT, to January 10, 1997, 02:00 UT. The panels from top to bottom are solar wind dynamic pressure; the IMF magnitude, $\theta$ and $\varphi ; \theta_{B n}$ at 11 and 12 MLT; $\Delta V$ measurements of the GEOTAIL and POLAR satellites. The dashed vertical line shows the start of the IMF fluctuations at WIND, and the dotted vertical line indicates the shock arrival at WIND.

\section{Magnetopause oscillations}

The magnetopause crossings by GEOTAIL appear as steep $N_{e}$ gradients, usually lasting 5-15 sec, although the outbound crossings tend to take a little longer $(5-25 \mathrm{sec})$. During these crossings, $\Delta V$ changes between 2 volts in the magnetosheath and $6-8$ volts in the LLBL/magnetosphere, corresponding to an $N_{e}$ change by a few 10's of electrons per $\mathrm{cm}^{3}$ or $N_{e}$ gradients of $0.5--2 \mathrm{~cm}^{-3}$ per sec. We applied minimum variance analysis to the magnetic field data during the crossings. The crossings are well-determined and the normals to the magnetopause are within 10 degrees of the predicted magnetopause normal angles [Fairfield, 1971], suggesting that the observed magnetopause motions simply reflect magnetosheath dynamic pressure changes.

The solar wind dynamic pressure does not correlate with the magnetopause oscillations in Figure 2, whereas the IMF orientation changes do correlate. The IMF orientation changes so that the dawnside bow shock varies frequently between quasiparallel and quasiperpendicular states in the intervals 14-16 UT and 20-23 UT, when boundary oscillations are observed. Note that in the interval 16-20 UT, when no proper magnetopause crossings are observed, the dawnside bow shock is mostly quasiperpendicular, suggesting that the magnetosheath pressure has increased during this time.

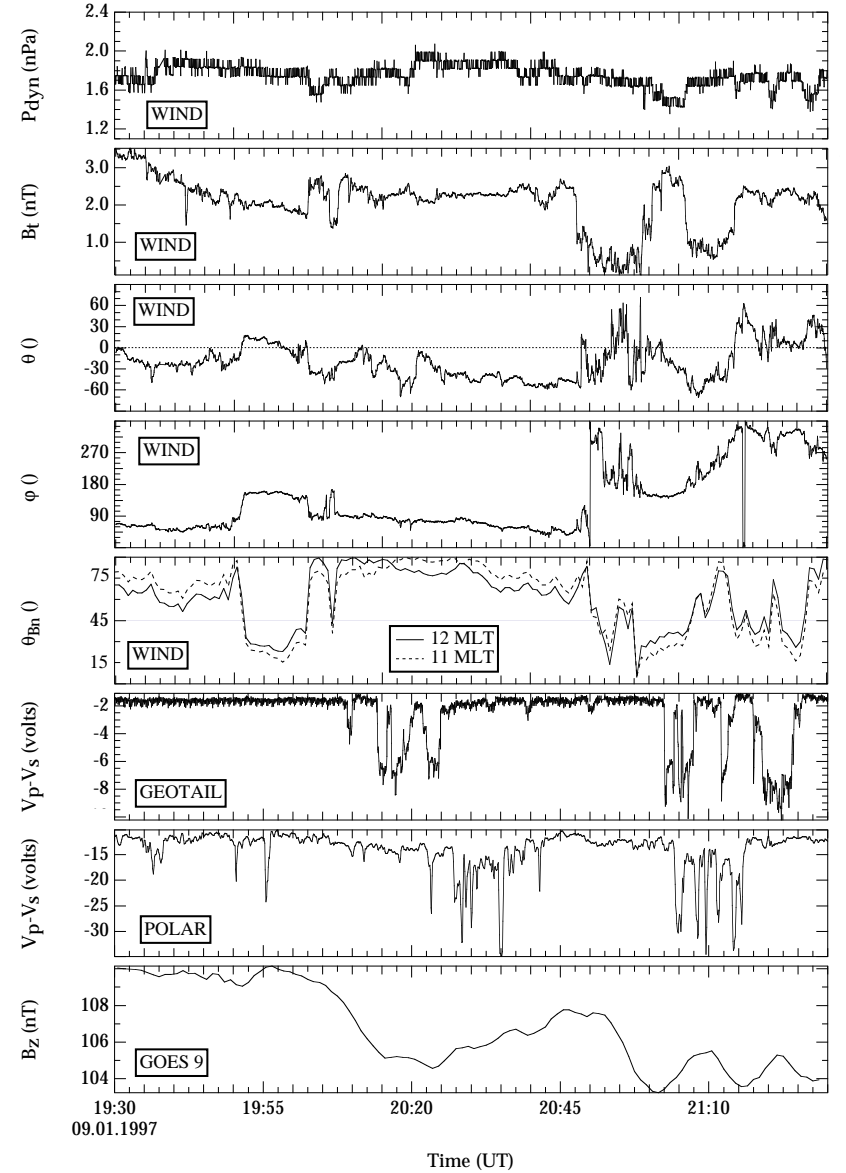

Figure 3. Two magnetopause oscillation events at 20:20 UT and 21:10 UT; similar events happened on POLAR with some delay. GOES 9, located at a geosynchronous orbit near noon, also observed weak disturbances. The top five panels from WIND suggest that plasma pressure does not vary, while the IMF has rapid directional changes.

Figure 3 displays a two-hour interval where two boundary oscillation events occur; the third and second panels from bottom present $\Delta V$ from GEOTAIL and POLAR, respectively. The bottom panel shows the northward component of the magnetic field from GOES 9 at geosynchronous orbit near noon. Decreases near 20:05 and 20:55 UT suggest reductions of magnetosheath pressure on the magnetopause which would cause outward movements of this boundary. These times are closely followed by magnetosheath to magnetosphere transitions by GEOTAIL (near the same meridian) and the related boundary motions observed by POLAR. The top five panels present the solar wind dynamic pressure and IMF data from WIND; the propagation time of a signal is expected to be about 15 minutes to the magnetopause at this moment. The most probable cause of these outward movements and oscillations of the magnetopause near 20:00 UT and 20:55 UT are the IMF $\varphi$ changes that produce a quasi-parallel bow shock in the pre-noon sector.

\section{Oscillations of the Plasma Mantle}

During the magnetopause oscillation in the interval 20$22 \mathrm{UT}$, the POLAR spacecraft is in the plasma mantle near 22-02 MLT and encounters the tenuous lobe several times 
as the mantle moves earthward/anti-earthward in response to the magnetosheath pressure variations. POLAR tends to observe the boundary motion with some delay with respect to GEOTAIL (see Figure 3). It is important to notice that the directions of the boundary motions at GEOTAIL and POLAR are in agreement: decreases in $\Delta V$ at GEOTAIL mean that the outward motion of the magnetopause is caused by reduced magnetosheath pressure. At this time the high-latitude boundary layer is also expected to move earthward (or sunward near 24 MLT) and POLAR should enter the lobe, as is observed. At 2010-2030 UT the boundary crossings of POLAR occur 7-11 minutes after those of GEOTAIL, while at 21-22 UT, the delay is only a few minutes. The actual length of delay is not important because it is strongly affected by the distance of the spacecraft from the boundary.

The $N_{e}$ gradients observed at the boundary between the mantle and the lobe are steep. Inbound and outbound crossings are quite similar; a typical crossing time is $25-40 \mathrm{~s}$ during which $\Delta V$ changed by $10-20$ volts or $0.2-0.6$ volts per s. This corresponds to an $N_{e}$ change from $0.5 \mathrm{~cm}^{-3}$ in the mantle to $0.1 \mathrm{~cm}^{-3}$ in the lobe. The crossing times suggest that the $N_{e}$ change occurs in a narrow transition layer which has a thickness of 1000-2000 km, assuming that the boundary moves at $50 \mathrm{~km} \mathrm{~s}^{-1}$; this speed is a typical value for fast magnetopause motion, which, however, may not be a representative value for the high-latitude boundary motion.

\section{Summary}

The magnetic cloud that arrived at the Earth at 01:05 UT on January 10, 1997 was preceded by a 12-hour interval of fluctuations in the IMF orientation which caused prolonged oscillations in the positions of the outer boundary of the Earth's magnetosphere. Both the $\operatorname{IMF} \varphi$ and $\theta$ vary, but it seems that the boundary oscillations are due to $\theta_{B n}$ variations. Specifically, the magnetosheath pressure decreased several times during quasiparallel shock conditions, which caused the expansion of the dayside magnetopause and the earthward motion of the high-latitude boundary layer.

Acknowledgments. We wish to thank S. Kokubun for the use of the GEOTAIL B-field data. H.S. was supported in part by NASA Interagency Order No. S-67019-F. H.L. was supported in part by an ISTP grant to the POLAR/EFI team at GSFC.

\section{References}

Anderson, B. J., ULF signals observed near the magnetopause, in Physics of the Magnetopause, edited by P. Song, B.U.Ö Sonnerup, and M.F. Thomsen, AGU Monograph 90, pp. 269276, 1995.

Fairfield, D. H., Average and unusual locations of the Earth's magnetopause and bow shock, J. Geophys. Res., 76, 6700-6716, 1971.
Fairfield, D. H. et al., Upstream pressure variations associated with the bow shock and their effects on the magnetosphere, J. Geophys. Res., 95, 3773- 3786, 1990. Upstream pressure variations associated with the bow shock and their effects on the magnetosphere, J. Geophys. Res., 95, 3773-3786, 1990.

Laakso, H. and A. Pedersen, Ambient electron density derived from differential potential measurements, in Measurement Techniques, edited by J. Borovsky, R. Pfaff, and Young, AGU Monograph, AGU, Washington, D.C., in press, 1997.

Lin Y., D. W. Swift, and L. C. Lee, Simulation of pressure pulses in the bow shock and magnetosheath driven by variations in interplanetary magnetic field direction, J. Geophys. Res., 101, 27251-27269, 1996.

Petrinec, S. M. and C. T. Russell, External and internal influences on the size of the dayside terrestrial magnetosphere, Geophys. Res. Lett., 20, 339- 342, 1993.

Phan, T.-D. et al., The magnetosheath region adjacent to the dayside magnetopause: AMPTE/IRM observations, J. Geophys. Res., 99, 121-141, 1994. magnetosheath region adjacent to the dayside magnetopause: AMPTE/IRM observations, $J$. Geophys. Res., 99, 121-141, 1994.

Phan, T.-D. and G. Paschmann, Low-latitude dayside magnetopause and boundary layer for high magnetic shear, 2. Structure and motion, J. Geophys. Res., 101, 7801, 1996.

Rosenbauer, H. et al., Heos 2 plasma observations in the distant polar magnetosphere: the plasma mantle, J. Geophys. Res., 80, 2723-2737, 1975. Sckopke, Heos 2 plasma observations in the distant polar magnetosphere: the plasma mantle, J. Geophys. Res., 80, 2723-2737, 1975.

Sibeck, D. G., R. E. Lopez, and E. C. Roelof, Solar wind control of the magnetopause shape, location, and motion, J. Geophys. Res., 96, 5489-5495, 1991.

Song, P., R. C. Elphic, and C. T. Russell, ISEE 1 and 2 observations of the oscillating magnetopause, Geophys. Res. Lett., 15, 744-747, 1988.

M. R. Collier, D. H. Fairfield, R. P. Lepping, R. F. Pfaff, Laboratory for Extraterrestrial Physics, NASA Goddard Space Flight Center, Greenbelt, MD 20771.

B. L. Giles, Space Science Laboratory, NASA Space Flight Center, Huntsville, Alabama 35812.

H. Laakso, H. Opgenoorth, Geophysical Research, Finnish Meteorological Institute, P.O. Box 503, Helsinki, 00101, Finland. (e-mail: harri.laakso@fmi.fi)

R. P. Lin, F. S. Mozer, T.-D. Phan, Space Sciences Laboratory, University of California, Berkeley, CA 94720.

D. G. Sibeck, The Johns Hopkins University, Applied Physics Laboratory, Laurel, MD 20723.

H. J. Singer, Space Environment Center, NOAA R/E/SE, 325 Broadway, Boulder, CO 80302.

K. Tsuruda, Institute of Space and Astronautical Science, Yoshinodai, Sagamihara, Kanagawa 299, Japan.

J. R. Wygant, School of Physics and Astronomy, University of Minnesota, Minneapolis, MN 55455.

(Received October 15, 1997; accepted January 15, 1998.) 